\title{
Multiplicative phase-shifting interferometry using optical flow
}

\author{
J. Vargas,${ }^{1, \star}$ J. Antonio Quiroga, ${ }^{2}$ C. O. S. Sorzano, ${ }^{1}$ J. C. Estrada,${ }^{3}$ and M. Servín ${ }^{3}$ \\ 'Biocomputing Unit, Centro Nacional de Biotecnología-CSIC, C/ Darwin 3, 28049, Cantoblanco (Madrid), Spain \\ ${ }^{2}$ Optics Department, Universidad Complutense de Madrid, Facultad de CC. Físicas, Ciudad Universitaria s/n, \\ 28040 Madrid, Spain \\ ${ }^{3}$ Centro de Investigaciones en Óptica A. C., Loma del Bosque 115, Col. Lomas del Campestre, 37150, \\ León (Guanajuato), Mexico \\ *Corresponding author: jvargas@cnb.csic.es
}

Received 30 April 2012; revised 23 July 2012; accepted 28 July 2012; posted 30 July 2012 (Doc. ID 167713); published 16 August 2012

\begin{abstract}
Fringe patterns with a multiplicative phase shift among them appear in experimental techniques as photoelasticity and RGB shadow moiré, among others. These patterns cannot be processed using standard phase-shifting demodulation techniques. In this work, we propose to use a multiframe regularized optical flow algorithm to obtain the interesting modulating phase. The proposed technique has been applied to simulated and experimental interferograms obtaining satisfactory results. (c) 2012 Optical Society of America
\end{abstract}

OCIS codes: $\quad 100.5070,100.2650,120.2650$.

\section{Introduction}

Phase-shifting interferometry (PSI) is an optical metrology technique for measuring the modulated phase of interferograms, using a controlled phase change or piston term between successive interferograms [1]. Other phase-shifting algorithms allow the determination of the modulating phase without any prior knowledge about the phase shifts (asynchronous detection) $[2,3]$. However, there are experimental techniques, such as photoelasticity or RGB shadow moiré, where it is experimentally difficult to introduce a phase shift in the modulating phase. In these cases, it is introduced a multiplicative phase shift. The modulating phase is multiplied by a different value-multiplicative phase shift-between successive interferograms. Therefore, the phase map is different for all interferograms and it does not correspond to a common two-dimensional function modified by different additive phase steps or piston terms.

$1559-128 \mathrm{X} / 12 / 245903-06 \$ 15.00 / 0$

(C) 2012 Optical Society of America
We can mathematically model these multiplicative phase-shifting interferograms as

$$
\begin{gathered}
I_{n}(x, y)=a(x, y)+b(x, y) \cos \left[\alpha_{n} \Phi(x, y)\right], \\
n \in[1, N],
\end{gathered}
$$

where $a(x, y)$ is the background illumination or DC component, $b(x, y)$ and $\Phi(x, y)$ are the modulation and phase maps, $\alpha_{n}$ are real-valued multiplicative phase shifts and $N$ is the number of fringe patterns. Note that, as mentioned above, we cannot demodulate the fringe patterns shown in (1) using phaseshifting techniques because the phase maps are different in all cases. Therefore, we need to implement other (non-phase-shifting) demodulating algorithms for this problem.

In the past there have been reported works about multiplicative PSI [4,5]. References [4,5] present techniques for demodulating multiplicative phaseshifting interferograms obtained from a photoelastic sample in a load-stepping experiment [4] and from a surface topography measurement by means of an 
RGB shadow moiré technique [5]. In both cases, the method is based on determining the sign of the quadrature signal $I_{Q}$ defined as $I_{Q}(x, y)=-\sin [\alpha \Phi(x, y)]$, where $\alpha$ is the multiplicative phase shift between two patterns. In order to obtain this sign, the first step is to normalize the fringe patterns, which consist in background suppression and modulation equalization. After this, we obtain the sign of $I_{Q}$ calculating the gradient of the normalized intensity with respect to $n$. Note that this method assumes that the phase is monotonic with respect to $n$ [4]. The monotonicity restriction limits the application of this method, and there are interesting problems that cannot be processed using this approach. Some examples are interferograms with an arbitrary phase variation between them, as deformation measurement in speckle interferometry or lens testing by moiré deflectometry [4]. Additionally, the performance of this method depends on the value of the phase variation between samples that must be high enough [4].

In this work, we propose a robust multiplicative PSI demodulating method based on a multiframe regularized optical flow approach (MOF). A two-step optical flow approach was previously applied in standard PSI to process two fringe patterns with an unknown phase step between them [6]. This previous method is herein extended to process several patterns with a multiplicative phase step between them that is not known. Observe that the effect that appears in multiplicative phase-shifting is a shrinking or a stretching in the fringe frequency between interferograms depending on $\alpha_{n} \geq 1$ or $\alpha_{n}<1$. These changes also provoke a movement of the fringes between successive fringe patterns that can be computed by the optical flow method and used to determine the direction map that is the same for all the interferograms. The proposed MOF method first obtains for each pattern, the direction map using a regularized optical flow approach between successive interferograms. After that, we acquire a reliable direction estimation from the previously obtained direction maps performing a weighted average. Observe that, although the interferograms have different modulating phases because the multiplicative phase step values, they all have the same direction map. Finally, the modulating phase can be obtained from the spiral phase transform (SPT) [7]. Note that this method is not affected by the monotonicity restriction as the method used in $[4,5]$ and it is also robust against noise because its regularizating character imposes continuity in the reconstructed phase. However, it is important to mention that the proposed method requires the phase difference between successive interferograms not to be higher than $\pi$ rad. If this requirement is not fulfilled, we will have additional fringes between successive interferograms and we will obtain a direction map affected by an ambiguity in its sign. Note that this is not a big concern as the multiplicative phase step is a variable that can be typically controlled experimentally. However, if we cannot control this magnitude and we have ambiguity in the direction map estimation, we can perform an additional regularization process to this direction map, imposing continuity, as we will see later. In these cases, we propose to use the direction regularization approach proposed in [8].

The paper is organized as follows: In Section 2, we present the theoretical foundations of the method. After this, in Section 3 , we give some numerical simulations to evaluate the accuracy and reliability of our technique. Then in Section $\underline{4}$, we present some experimental results obtained from loading-stepping photoelasticity and shadow moiré techniques. We finish with the conclusions of the presented work.

\section{Theoretical Foundations}

The optical flow approach is a standard method in computer vision for obtaining the distribution of apparent velocities of objects, surfaces, and edges in an image set [9]. Suppose we have two images obtained at times $t$ and $t+\Delta t$ such that there is a local motion between them. Note that this effect can appear because the observed object (in our case the fringe pattern) or the camera moves along time. We assume that corresponding pixels between both images have approximately the same intensity-brightness constancy assumption. Therefore, an arbitrary pixel at time $t$ given by $(x, y, t)$ and with intensity $I(x, y, t)$ will move to a new position $(x+\Delta x, y+\Delta y, t+\Delta t)$ with intensity $I(x+\Delta x, y+\Delta y, t+\Delta t)$, where $I(x, y, t)=$ $I(x+\Delta x, y+\Delta y, t+\Delta t)$. Observe that the pixels given by $(x, y, t)$ and $(x+\Delta x, y+\Delta y, t+\Delta t)$ are corresponding pixels as they are observing the same object point at different times. Assuming the movement is small we can expand the intensity map at $t+\Delta t$ as

$$
\begin{aligned}
I(x+\Delta x, y+\Delta y, t+\Delta t) \cong & I(x, y, t)+\frac{\partial I}{\partial x} \Delta x \\
& +\frac{\partial I}{\partial y} \Delta y+\frac{\partial I}{\partial t} \Delta t .
\end{aligned}
$$

As mentioned earlier, we suppose that the brightness of a point does not change along timebrightness constancy assumption-therefore, $I(x+$ $\Delta x, y+\Delta y, t+\Delta t)=I(x, y, t)[\underline{9}]$ and Eq. (2) can be rewritten as

$$
\frac{\partial I}{\partial x} u+\frac{\partial I}{\partial y} v+\frac{\partial I}{\partial t}=0,
$$

where $u=\frac{\partial x}{\partial t}$ and $v=\frac{\partial y}{\partial t}$ are the velocity components and correspond to $u \stackrel{\partial t}{=} \Delta x / \Delta t$ and $v=\Delta y / \Delta t$. For the sake of clarity, we have omitted the spatial dependences in the deduction shown above. Observe that $\Delta x, \Delta y, u$, and $v$ are scalar fields, as the movement of the different pixels can be different, and then, we have that $\Delta x(x, y), \Delta y(x, y) u(x, y)$, and $v(x, y)$. Eq. (4) introduces a restriction in $u$ and $v$ at every pixel, but as we need to obtain two variables, $u(x, y)$ and $v(x, y)$, we need to introduce at least one additional constraint. One possibility is to impose 
smoothness in the $u$ and $v$ fields using a regularized optical flow method [9]. The modulating phase must therefore belong to the topological space of smooth continuous functions. We can define the following energy functional [9]:

$$
E^{2}=\left(I_{x} u+I_{y} v+I_{t}\right)^{2}+\mu\left(u_{x}^{2}+u_{y}^{2}+v_{x}^{2}+v_{y}^{2}\right),
$$

where $I_{j}=\partial I / \partial j, \quad u_{j}=\partial u / \partial j, \quad v_{j}=\partial v / \partial j \quad$ with $j=[x, y] ; E$ is the energy to be minimized; and $\mu$ is the regularizing parameter that weighs the smoothness in $u$ and $v$. We can obtain the regularized $u$ and $v$ components for every pixel minimizing Eq. (4) using the Gauss-Seidel iterative solution [9] as

$$
\begin{aligned}
& u^{k+1}=\bar{u}^{k}-I_{x}\left[I_{x} \bar{u}^{k}+I_{y} \bar{v}^{k}+I_{t}\right] /\left(\mu^{2}+I_{x}^{2}+I_{y}^{2}\right) \\
& v^{k+1}=\bar{v}^{k}-I_{y}\left[I_{x} \bar{u}^{k}+I_{y} \bar{v}^{k}+I_{t}\right] /\left(\mu^{2}+I_{x}^{2}+I_{y}^{2}\right),
\end{aligned}
$$

where $u^{k+1}$ and $v^{k+1}$ are the velocity components obtained in the iteration $k+1$, with $k$ a positive integer and $\bar{u}$ and $\bar{v}$ correspond to the mean value of $u$ and $v$ in a defined neighborhood $\Omega$. The region $\Omega$ is centered at pixel $(x, y)$. The minimization procedure is stopped when a maximum number of iterations is reached or when a minimum energy value is obtained. Both parameters are input arguments of the algorithm and must be provided. Once we have obtained $u$ and $v$ maps at every pixel we can obtain the direction map as

$$
\theta=\arctan \left(\frac{v}{u}\right)
$$

If we have an interferogram sequence as shown in Eq. (1), we can obtain a direction map set computing $\theta_{n}$ between successive interferograms $I_{n}$ and $I_{n+1}$. Note that from $I_{n}$ and $I_{n+1}$ we will acquire the velocity components $u_{n}$ and $v_{n}$. As mentioned above, the different interferograms have different modulating phases because of the multiplicative phase steps. However, the direction maps $\theta_{n}$ have to be equal in all cases. Therefore, we can combine all the obtained direction maps in order to obtain an improved direction map estimation $\theta$ by a weighted average using as the quality or weigh map the following term [10]:

$$
Q_{n}(x, y)=\sqrt{u_{n}(x, y)^{2}+v_{n}(x, y)^{2}} .
$$

And then, taking into account that $\theta_{n}$ are discontinuous signals, we calculate the improved direction map $\theta$ from

$$
\begin{aligned}
\cos (\theta) & =\sum_{n} \cos \left(\theta_{n}\right) Q_{n}, \\
\sin (\theta) & =\sum_{n} \sin \left(\theta_{n}\right) Q_{n}, \\
\theta & =\arctan \left(\frac{\sin (\theta)}{\cos (\theta)}\right) .
\end{aligned}
$$

Obviously, if any direction map $\theta_{n}$ is affected by sign ambiguity because the phase difference between successive interferograms $I_{n}$ and $I_{n+1}$ is higher than $\pi \mathrm{rad}$, Eq. (8) will not provide an accurate direction map estimation $\theta$. We can use as reference $\theta_{1}$ assuming that this map is not affected by sign ambiguity and compare each $\theta_{n}$ with $n>1$ with respect $\theta_{1}$. We can impose that $Q_{n}$ is equal to zero in regions where $\sqrt{\left(\cos \left(\theta_{1}\right)-\cos \left(\theta_{n}\right)\right)^{2}+\left(\sin \left(\theta_{1}\right)-\sin \left(\theta_{n}\right)\right)^{2}}>1$. Therefore, we only have to assure that $\theta_{1}$ is not affected by sign ambiguity. If we cannot guarantee this point, we propose to use the fast direction regularization approach proposed in [8] to solve the sign ambiguity in $\theta_{1}$.

Once we obtain the regularized direction map, we can obtain the phase map using the SPT operator [7]. If we first subtract the DC component of $I_{n}$ in Eq. ( $\left.\underline{1}\right)$ by a typical high-pass filter and we obtain

$$
\operatorname{SPT}\left\{\tilde{I}_{n}\right\}=i \exp (i \theta) b \sin \left(\alpha_{n} \Phi\right),
$$

with $\tilde{I}_{n}=b \cos \left(\alpha_{n} \Phi\right)$. From Eq. (7) we obtain the quadrature signal as

$$
b \sin \left(\alpha_{n} \Phi\right)=-i \exp (-i \theta) \operatorname{SPT}\left\{\tilde{I}_{n}\right\},
$$

and finally, we obtain the modulating wrapped phase as

$$
W\left\{\Phi_{n}\right\}=W\left\{\alpha_{n} \Phi\right\}=\arctan \left(\frac{b \sin \left(\alpha_{n} \Phi\right)}{\tilde{I}_{n}}\right),
$$

where $W\{\cdot\}$ is the wrapping operator. We have, therefore, $N$ wrapped modulating phase maps affected by different and unknown multiplicative phase shifts $\alpha_{n}$. We first unwrap the different phases using the fast and robust against noise algorithm presented in [11]. Finally, we obtain a robust phase map estimation against noise by combining $\Phi_{n}$ as

$$
\tilde{\alpha} \Phi=\sum_{n} \Phi_{n} / N
$$

where $\tilde{\alpha}=\sum_{n} \alpha_{n} / N$. If we assume that $\alpha_{1}=1$, we finally obtain $\Phi$ from

$$
\tilde{\alpha}=\left(\sum_{x} \sum_{y} \tilde{\alpha} \Phi /\left(N_{x} N_{y} \alpha_{1} \Phi\right)\right), \Phi=\tilde{\alpha} \Phi / \tilde{\alpha},
$$

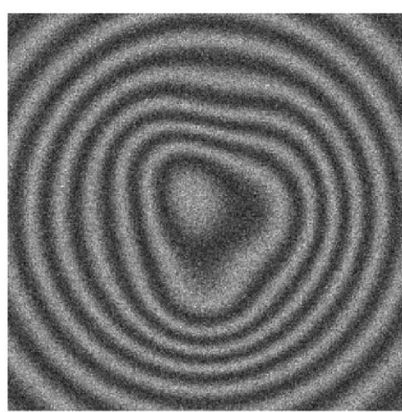

(a)

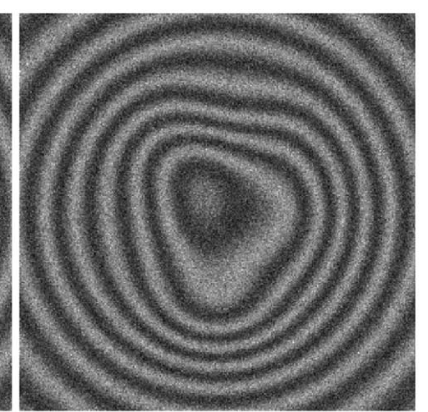

(b)
Fig. 1. Simulated fringe patterns used in the numerical analysis section. 


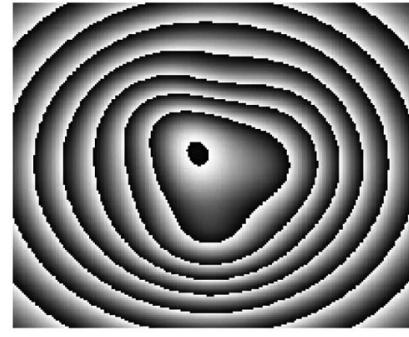

(a)

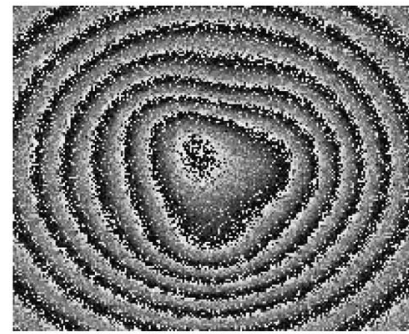

(c)

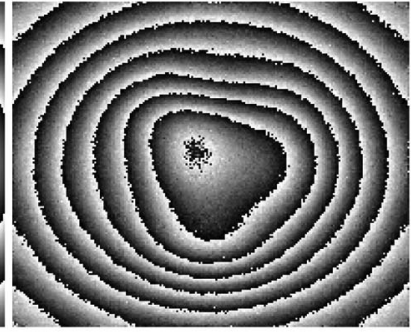

(b)

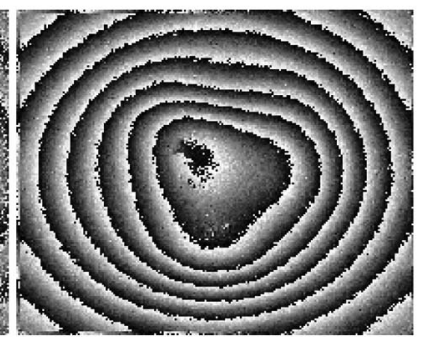

(d)
Fig. 2. (a) Ground truth modulating phase and retrieved phases using (b) the proposed MOF method, by the (c) TDM and by the (d) OVFR method.

with $N_{x}$ and $N_{y}$ the number of image columns and rows, respectively.

\section{Numerical Analysis}

We have performed some numerical analyses to study the performance of the proposed MOF algorithm in different cases. We have analyzed the method performance when we process different numbers of patterns, levels of noise, and different multiplicative phase steps. We have compared the results obtained by the MOF approach with the results obtained by the temporal demodulation method (TDM) $[\underline{4}, 5]$ and by the orientational vector field regularized estimator (OVFR) [9]. Note that the TDM and OVFR methods are limited to use two and one fringe patterns, respectively. In Fig. 1 we show two fringe patterns with a multiplicative phase shift between them 0.98 . The images size is $311 \times 311$ pixels. The noise is Gaussian and additive, and the signal-to-noise ratio (SNR) is of $6.25 \%$. In Fig. 2, we show the reference theoretical wrapped phase, and the obtained phases

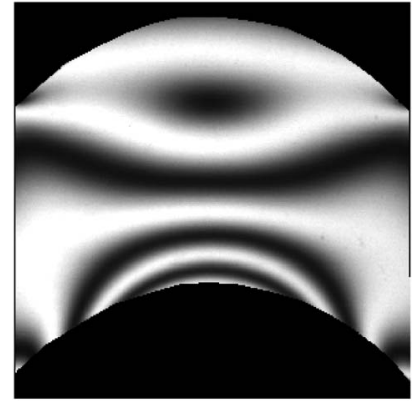

(a)

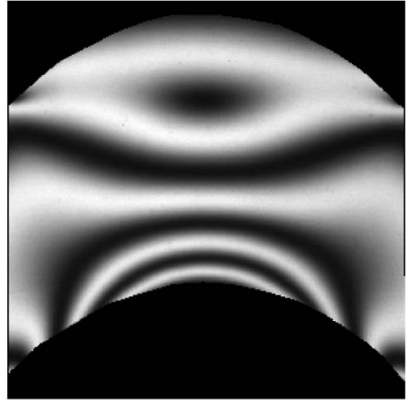

(b)
Fig. 4. Two experimental fringe patterns obtained in a loadingstepping photoelastic experiment.

by the proposed (a) MOF, (b) the TDM, and (c) the OVFR methods. Observe in Fig. 2 that the wrapped phase obtained by the TDM and the OVFR is affected by noise and nonlinearities. The rms errors of the difference between the theoretical and obtained unwrapped phases and the processing times are 0.30 , 1.23 , and $0.50 \mathrm{rad}$, and $1.10,0.01$, and $3.82 \mathrm{~s}$ when processing by the MOF, TDM, and OVFR methods, respectively with a $2.67 \mathrm{GHz}$ laptop and using MATLAB. In Fig. 3 we show the results obtained by the MOF, TDM, and OVFR methods when processing patterns with different levels of noise, multiplicative phase shifts, and, in the case of the MOF approach, different numbers of patterns. In Fig. 3, the different methods process patterns as similar to the ones shown in Fig. 1. In Figs. 3(b) and 3(c), the noise level that we use is 0.3 and 0.5 , respectively, which corresponds to a SNR of $2.2 \%$ and $6.25 \%$. From Fig. 3 we see that in all cases, the proposed MOF method obtains more accurate results than the TDM and OVFR approaches. Note also that in Fig. 3(c), the TDM and OVFR approaches only process two and three patterns, respectively. Additionally, we can see from Fig. 3(c) that the rms errors obtained by the MOF method when we process two and 15 patterns are $0.29 \mathrm{rad}$ and $0.11 \mathrm{rad}$, which represents an improvement ratio of approximately 2.6 .

\section{Experimental Results}

We have also applied the proposed algorithm to experimental fringe patterns obtained by load-stepping

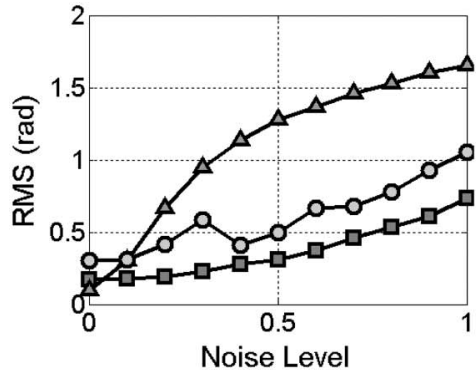

(a)

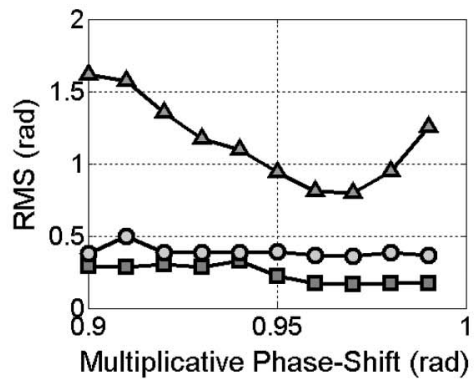

(b)

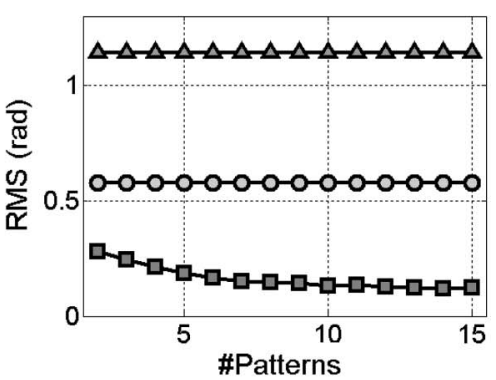

(c)

Fig. 3. Root mean square errors (RMS) obtained by the proposed method (solid black curve with dark squares), the TDM (solid black curve with gray triangles), and the OVFR method (solid black curve with gray circles) for (a) different levels of noise, (b) multiplicative phase-shifting, and (c) number of fringe patterns. 


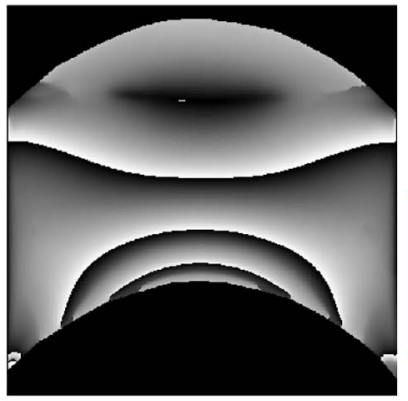

(a)

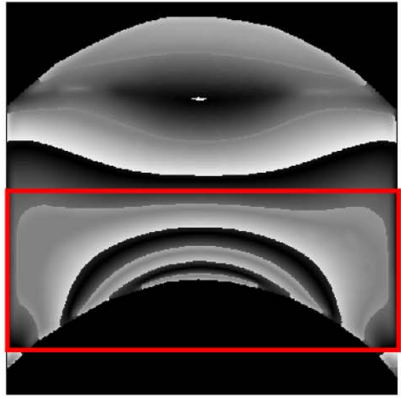

(b)

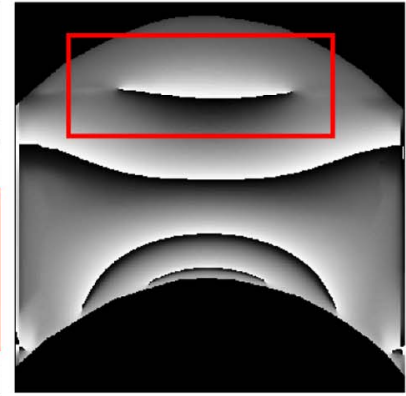

(c)

Fig. 5. (Color online) Obtained phases using (a) the proposed MOF method, (b) the TDM, and (c) the OVFR methods.

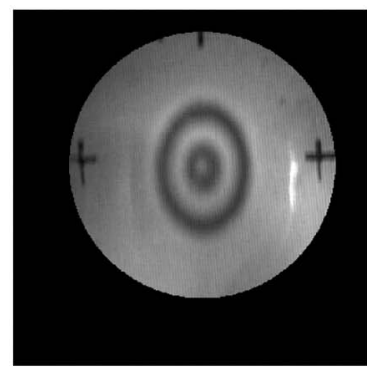

(a)

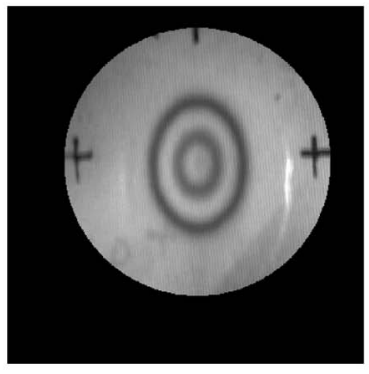

(b)

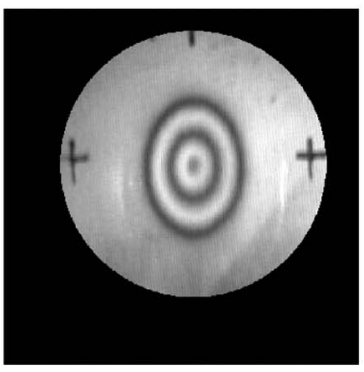

(c)

Fig. 6. Experimental fringe patterns obtained from an aeronautical surface with an indentation in an RGB shadow-moiré experiment.

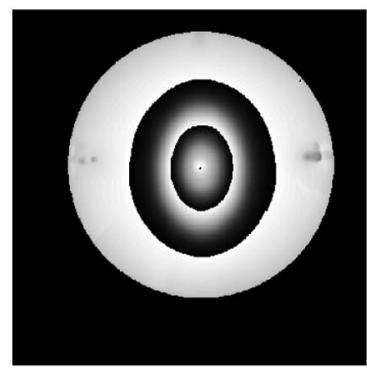

(a)

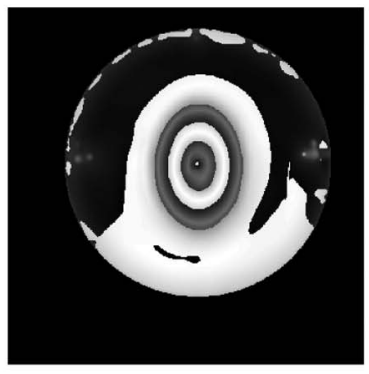

(b)

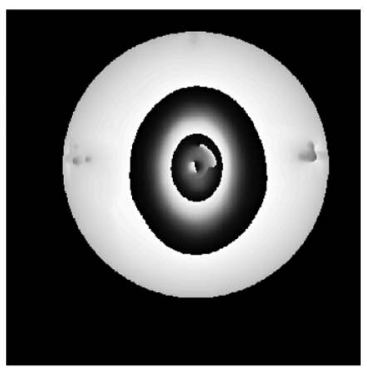

(c)

Fig. 7. Obtained phases using (a) the proposed MOF method, (b) the TDM, and (c) the OVFR methods.

photoelasticity and RGB shadow moiré approaches. As in the simulation section, we have compared the retrieved phases obtained from the proposed MOF method using three patterns, the TDM approach $[4,5]$ using two patterns, and the OVFR demodulating technique [9] using a single frame. In a loadstepping experiment, we measure the isochromatic fringe pattern of a photoelastic sample as the applied load changes. Therefore, we obtain a temporal sequence of multiplicative phase-shifting interferograms. Note that in this experiment the amount of load-step and then of multiplicative phase-shifting is controlled experimentally. Additionally, in an RGB shadow moiré experiment, we are interested in measuring the topography of a surface by means of three LEDs of different colors that illuminate and project a grating onto the surface under study. We obtain a polychromatic fringe pattern composed by a mixture of three multiplicative phase-shifting interferograms. In this case, the different positions of the light sources, which can be controlled experimentally, determine the different multiplicative phase steps.

In Fig. 4 we show two multiplicative phase-shifting patterns obtained from a load-stepping photoelasticity experiment. The images have a size of $574 \times 768$. In Fig. 5, we show the phases obtained by (a) the MOF, (b) the TDM, and (c) the OVFR methods. The obtained multiplicative phase steps are $\alpha_{1}=1$ and $\alpha_{2}=0.95$. We can see in Figs. 5(b) and 5(c) two marked regions with red rectangles where visually the TDM and OVFR approaches cannot obtain accurate results. In the case of the TDM method we can see that high nonlinearities appear in the reconstructed phase. On the other hand, in case of the OVFR we can see that a discontinuity appears in the wrapped phase. The required processing times are $3.8,0.04$, and $14.9 \mathrm{~s}$ when processing by the MOF, TDM, and OVFR methods, respectively. 
In Fig. 6, we show three fringe patterns obtained by an $R G \bar{B}$ shadow moiré technique when an aeronautical surface with an indentation is observed. In Fig. 6 , we have masked the region of interest where the indentation appears. Observe from Fig. 6 that the fringe patterns are affected by a multiplicative phase step. Finally, in Fig. 7 we show the resultant obtained wrapped phases by (a) the MOF, (b) the TDM, and (c) the OVFR approaches. As in the case before, we can observe visually that the phase retrieved by the TDM method has low accuracy because it is highly affected by nonlinearities. Additionally, the phase obtained by the OVFR method present discontinuities in the central part of the phase map. In this case, the obtained multiplicative phase steps are $\alpha_{1}=1, \alpha_{2}=0.94$, and $\alpha_{3}=0.98$.

\section{Conclusions}

In conclusion, we have presented a multiplicative and multiframe phase-shifting method for demodulating interferograms with sensitivity change. The proposed method is based on a regularized optical flow method that can combine several multiplicative phase-shifting interferograms. The multiplicative phase shifts between interferograms has not to be known. Note that this method is not affected by the monotonicity restriction as the method used in $[\underline{4}, \underline{5}]$ and it is also robust against noise because of its regularizing character. We have tested the proposed method with simulated and real interferograms. We have compared our algorithm with the TDM $[\underline{4}, \underline{5}]$ and the OVFR [9] methods and we have shown a very good performance of the proposed method in terms of accuracy.

\section{References}

1. D. Malacara, M. Servín, and Z. Malacara, Interferogram Analysis for Optical Testing (Cambridge University, 2004).

2. Z. Wang and B. Han, "Advanced iterative algorithm for phase extraction of randomly phase-shifted interferograms," Opt. Lett. 29, 1671-1673 (2004)

3. J. Vargas, J. Antonio Quiroga, and T. Belenguer, "Phaseshifting interferometry based on principal component analysis," Opt. Lett. 36, 1326-1328 (2011).

4. J. A. Quiroga, J. A. Gómez-Pedrero, M. J. Terrón-López, and M. Servín, "Temporal demodulation of fringe patterns with sensitivity change," Opt. Commun. 253, 266-275 (2005).

5. J. A. Gómez-Pedrero, J. A. Quiroga, M. J. Terrón-López, and D. Crespo, "Measurement of surface topography by RGB shadow-moiré with direct phase demodulation," Opt. Lasers Eng. 44, 1297-1310 (2006).

6. J. Vargas, J. A. Quiroga, C. O. S. Sorzano, J. C. Estrada, and J. M. Carazo, "Two-step interferometry by a regularized optical flow algorithm," Opt. Lett. 36, 3485-3487 (2011).

7. K. G. Larkin, D. J. Bone, and M. A. Oldfield, "Natural demodulation of two-dimensional fringe patterns. I. General background of the spiral phase quadrature transform," J. Opt. Soc. Am. A 18, 1862-1870 (2001).

8. J. Villa, I. De la Rosa, G. Miramontes, and J. A. Quiroga, "Phase recovery from a single fringe pattern using an orientational vector-field-regularized estimator," J. Opt. Soc. Am. A 22, 2766-2773 (2005).

9. B. K. P. Horn and B. G. Schunck, "Determining optical flow," Artif. Intell. 17, 185-203 (1981).

10. B. Ströbel, "Processing of interferometric phase maps as complex-valued phasor images," Appl. Opt. 35, 2192-2198 (1996).

11. M. Arevallilo-Herráez, D. R. Burton, M. J. Lalor, and M. A. Gdeisat, "Fast two-dimensional phase-unwrapping algorithm based on sorting by reliability following a noncontinuous path," Appl. Opt. 41, 7437-7444 (2002). 